Review

\title{
The Immune Privilege of the Intervertebral Disc: Implications for Intervertebral Disc Degeneration Treatment
}

\author{
Zhen Sun"1\#, Bing Liu²\#, Zhuo-Jing Luo ${ }^{\circledR}$ \\ 1. Department of Orthopedic, Xijing Hospital, Fourth Military Medical University. Western Changle Road, Xi'an, 710032, Shannxi Provence, P. R. China \\ 2. Department of Radiology, Xijing Hospital, Fourth Military Medical University. Western Changle Road, Xi'an, 710032, Shannxi Provence, P. R. China. \\ \#These authors contributed equally to this work. \\ $\triangle$ Corresponding author: Zhuo-Jing Luo. E-mail: zjluo@fmmu.edu.cn; FAX: +86 29 84775285; Phone: +86 2984775285. \\ (c) The author(s). This is an open access article distributed under the terms of the Creative Commons Attribution License (https://creativecommons.org/licenses/by/4.0/). \\ See http://ivyspring.com/terms for full terms and conditions.
}

Received: 2019.11.18; Accepted: 2020.01.29; Published: 2020.02.24

\begin{abstract}
The intervertebral disc (IVD) is the largest avascular organ of the body. It is composed of three parts: the nucleus pulposus (NP), the annulus fibrosus (AF) and the cartilaginous endplate (CEP). The central NP is surrounded by the AF and sandwiched by the two CEPs ever since its formation. This unique structure isolates the NP from the immune system of the host. Additionally, molecular factors expressed in IVD have been shown inhibitive effect on immune cells and cytokines infiltration. Therefore, the IVD has been identified as an immune privilege organ. The steady state of immune privilege is fundamental to the homeostasis of the IVD. The AF and the CEP, along with the immunosuppressive molecular factors are defined as the blood-NP barrier (BNB), which establishes a strong barrier to isolate the NP from the host immune system. When the BNB is damaged, the auto-immune response of the NP occurs with various downstream cascade reactions. This effect plays an important role in the whole process of IVD degeneration and related complications, such as herniation, sciatica and spontaneous herniated NP regression. Taken together, an enhanced understanding of the immune privilege of the IVD could provide new targets for the treatment of symptomatic IVD disease. However, the underlying mechanism above is still not fully clarified. Accordingly, the current study will extensively review and discuss studies regarding the immune privilege of the IVD.
\end{abstract}

Key words: intervertebral disc, nucleus pulposus, annulus fibrosus, immune privilege, auto-immune response

\section{Introduction}

Intervertebral disc (IVD) degeneration is one of the most common contributors to spinal degenerative disease, which leads to individual sufferings such as acute/chronic pain, disability and psychological problems, causing enormous social and economic burden $[1,2]$. Current strategies for IVD degeneration management include conservative and surgical treatment [3]. Recently, biological options, such as biomaterials application, cell translation and genetic modification, have shown promising beneficial effects on IVD regeneration [4-7]. However, the pathologi- cal process of IVD degeneration is still not fully understood.

As the largest avascular organ of the body, the IVD sits between the vertebras and is responsible for the support, durability and flexibility of the spine [8]. Anatomically, the IVD is a complex tissue comprising of three parts: a central proteoglycan-rich core, the nucleus pulposus (NP), an outer circumferential ring of fibrocartilage, the annulus fibrosus (AF), and the two cartilaginous endplates (CEP) adjoining the vertebra bodies [9]. The gelatinous NP is composed of cell clusters embedded in a proteoglycan-collagen- 
rich extracellular matrix (ECM). Ever since its formation, the NP tissue has been trapped in the IVD by the surrounding $\mathrm{AF}$ and $\mathrm{CEP}$, and this unique structure isolates the NP from the immune system of the host. Meanwhile, various ingredients of the NP were found to cause auto-immune reactions after exposure to the host immune system during IVD degeneration progress [10, 11]. For this reason, the IVD has been identified as an immune privilege organ. In fact, these features have long been observed and the concept of IVD immune privilege has been proposed. Although the immune privilege status is essential for IVD homeostasis and normal function, there have been no studies summarizing the state-ofthe-art in this field until now. Therefore, the current study will extensively review and discuss relative studies regarding the immune privilege of the IVD.

\section{The development of the IVD}

The IVD is derived from embryonic structures termed as the sclerotome and notochord between the developing vertebrae. With the formation of vertebrae, sclerotome condenses around the notochord to form the vertebrae and the putative AF. At the same time, notochordal is contracted from the vertebral body and expands into the area of the future NP. The notochordal is compressed and become entrapped in a dense ring of sclerotome-derived connective tissue. The centralized notochordal element develops to the NP tissue in the early fetal life and grows rapidly in the late fetal life and early infancy. On the other hand, the surrounding dense ring of sclerotome-derived connective tissue develops to the AF with abundant
ECM containing collagens and glycoproteins. Meanwhile, chondrocyte differentiation and endochondral bone formation occur in the vertebral bodies with high concentrations of bone morphogenetic protein (BMP) activity [12]. When the bony vertebra is formed, hyaline cartilage adjacent to the IVD is maintained and develops to the CEP at the end plate (Figure 1). In the early stage of human life, the NP is populated by clusters of large, vacuolated notochordal cells and by small chondrocyte-like cells [13]. However, by the second decade of life, the notochordal cells in the NP disappear, and the NP transitions from a notochordal structure to a tissue embedded with small chondrocyte-like cells [14]. It is speculated that early IVD degeneration happens with the disappearance of notochordal cells [15]. During this process, it is noteworthy that the NP tissue is sealed and isolated from the immune system ever since its formation at the beginning of IVD development.

\section{The blood-NP barrier}

Immune privilege organs are operationally defined as regions in the body where foreign tissue grafts can survive and extend with indefinite periods of time, while similar implants placed at regular regions of the body are acutely rejected. Well studied structures include the eye [16] and the brain [17], along with the testis [18] and pregnant uterus [19]. In these structures, barriers between the internal environment and the host immune system have been widely observed and explored in-depth. However, the mechanism of NP-host immune system isolation is still undefined.
A

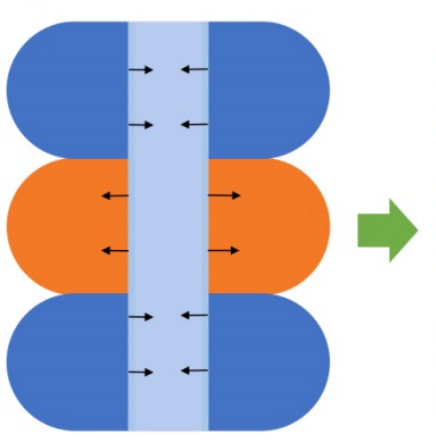

B

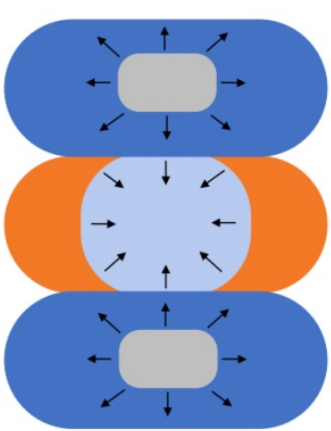

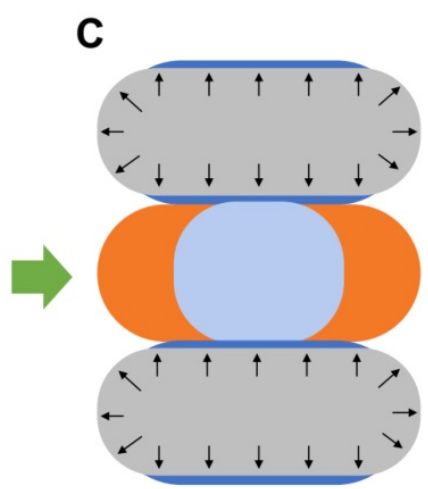

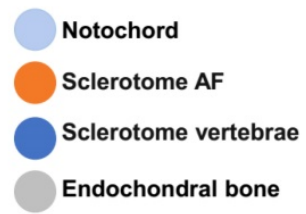

Figure 1. Development of the intervertebral disc (IVD). A. Sclerotome condenses around the notochord to form the vertebrae and the putative annulus fibrosus (AF) while the notochordal is contracted from the vertebral body and expands into the area of the future nucleus pulposus (NP) area. B. The notochordal is compressed and becomes entrapped in the surrounding dense ring of sclerotome-derived connective tissue, which develops to the AF. C. Endochondral bone formation occurs in the sclerotome vertebra and expands to become bony vertebra. Hyaline cartilage adjacent to the IVD is maintained and develops to the cartilaginous endplate (CEP). 
Accumulating evidence has suggested the existence of machinery that limits immunocytes and immune mediators entering the NP tissue in IVD. This machinery, here, could be defined as a blood-NP barrier (BNB), which is a complex composition of physical and molecular factors. In anatomical view, the $\mathrm{BNB}$ is a region that isolates the NP tissue from the host immune system. It is generally composed of the AF and the CEP. The AF is composed of 15-25 concentric layers, which consists of alternatingly aligned oblique collagen fibers interspersed with proteoglycans. This feature holds the NP by confine its swelling pressure and resisting shear and tensile stresses from the internal pressure exerted by the NP [12]. The CEP is a layer of cartilage with about 0.5-1 $\mathrm{mm}$ thickness. It contains ECM of aggrecan and collagens with chondrocytes embedded in it [20]. The CEP serves not just as an interface between the soft $\mathrm{NP}$ and the dense bone of the vertebrae, but as a physical barrier that prevents immune cells and cytokines to the bone. In addition, studies have shown that the high proteoglycan concentration, together with micro-environment of high physical pressure, inhibits the ingrowth of blood vessels [21], which act as significant channel of immunocytes infiltration. Therefore, the AF and the CEP constitute a strong basement isolating the NP tissue from the host immune system.

On the other hand, pilot studies have found the inhibition effect of molecular factors expressed in IVD and indicated their important role in the long-term maintenance of IVD immune privilege. Studies showed that Fas ligand (FasL), which is an apoptosis inducer and widely expressed in other immune privilege sites, exists in human NP tissues [22, 23]. In our previous studies, we found that FasL could induce apoptosis of both vascular endothelial cells and immunocytes including macrophages and $\mathrm{CD} 8^{+} \mathrm{T}$ cells $[24,25]$. These studies indicate that FasL might act as a molecular barrier by eliminating blood vessel infiltration and immune cells recruitment. In another study, Wiet et al. found that healthy AF conditioned medium obtained from AF cultures could inhibit mast cell activation by downregulating its expression of vascular endothelial growth factor (VEGF), tumor necrosis factor (TNF)- $\alpha$, interleukin (IL)-1 $\beta$ and chemokine (C-C motif) ligand 2 (CCL2/MCP-1), and inhibit mast cell induced angiogenesis [26]. In addition, numerous studies have reported the protective effect of notochordal cells in IVD and its suppressive impact on inflammation [27, 28]. Cornejo et al. and Kwon et al. found soluble factors from notochordal cells inhibit endothelial cell invasion and vessel formation by suppressing VEGF signaling [29, 30]. Meanwhile, Purmessur et al. found intact glycos- aminoglycans from IVD-derived notochordal cellconditioned media inhibit neurite growth while maintaining neuronal cell viability [31]. Yet, in the study of de Vries et al., the anti-angiogenic and antineurogenic effects of notochordal cell-conditioned media were not observed [32]. This is, as the authors pointed, attributed to the different life stages and breeds of sacrificed animals, and cell culture conditions. However, evidence is still limited in the interaction between notochordal cells and immunocytes, and the role of notochordal cells in IVD immune privilege needs more studies.

Collectively, current studies are still limited in the immunosuppressive effects of molecular factors in IVD. The exact molecules mediating these effects are still not fully understood. Multiple factors such as exosomes and miRNAs might be involved and more studies are needed to clarify the mechanism in this aspect. Altogether, the AF, the CEP and molecular factors such as FasL, establish a unique architecture for immune privilege resembling that of a medieval castle (Figure 2A).

\section{Auto-immune response of the NP}

In IVD degeneration, BNB damage such as fissure and tear is commonly observed as pathological change in the AF and CEP $[33,34]$. It has been shown that fissure of $\mathrm{AF}$ is mechanically and chemically conducive to the ingrowth of blood vessels [35]. The auto-immune response and downstream cascade reaction starts when the BNB is damaged. In fact, as early as the 1960s, studies have found the evidence of auto-immune response of the degenerated NP in patients and animals [36, 37], and indicated that radicular pain of a lumbar disc herniation results from chemicals of exposure of the NP and related auto-immune response [38]. Following studies were conducted trying to explore this phenomenon. In particular, Satoh et al. studied eight patients with lumbar disc herniation and found antigen-antibody complexes seem to be commonly present in the herniated NP tissue [39]. To identify the immunocyte types, Geiss A et al. found activated T and B cells were elevated by autologous NP subcutaneously in an animal model [40]. In another study, they found that $\mathrm{T}$ cells could be activated by autologous NP tissue simulation [41]. Murai et al. found that macrophages and NK cells could recognize autologous NP cells and showed positive cytotoxic effects by comparation of wild type mice and immune-deficient mice [42]. Moreover, Geiss A et al. found predominately plasmacytoid dendritic cells (PDCs) along with few macrophages and memory $\mathrm{T}$ cells in both sequestrated and extruded discs, suggesting that (PDCs) play an important role in initiation of an immune 
response of NP while macrophages may mediate disc resorption at a later stage [43]. Most recently, Lee et al. showed macrophage infiltration into injured IVD along with pathological innervation in long-term histological analysis of punctured mice IVD model [44]. In canine IVD herniation, Monchaux et al. found that monocytes and macrophages existed in extruded IVD material [45]. These findings suggested complicated types of immunocytes involve in the autoimmune response of the NP in different stages and macrophages might play an important role in this pathological process. More studies are needed to explore the underly mechanism of their roles.

The direct evidence of the NP autoimmune response was found by Capossela et al., who identified IgGs against collagen type I, II, V, and aggrecan in human degenerated IVD samples [10]. Moreover, inflammatory factors were found to have increased expression in the study of Takada et al, who found IVD autografts induced TNFa, IL-6 and IL-8; as well as cyclooxygenase 2 up-regulation and macrophage infiltration in sciatica [46]. By co-culture of autologous or allogeneic peripheral blood mononuclear cells and NP cells, Stich et al. found elevated immune cell proliferation levels in 3Dcultures than 2D-cultures, and a general trend to higher responses for NP cells from severely degenerated IVD tissue [47], indicating that autoimmune response could vary depending on different cell cultures and degeneration degrees. More recently, Silva et al. established a model of IVD organ culture, found that human macrophages could be polarized toward a more pro-inflammatory profile by degenerated IVD tissue, and interfere with IVD ECM remodeling by downregulating aggrecan and collagen II gene expression in the presence of IL-1 $\beta$ [48]. These findings showed that with the breakdown of the BNB, the exposed NP tissue could induce autoimmune response, which stimulates both immunocytes activation and inflammatory factors infiltration (Figure 2B).

Meanwhile, the recruitment of immunocytes could lead to the deterioration of IVD degeneration via cell-cell biocommunication and cytokines secretion. As for the IVD cells, studies have showed altered phenotypes and function in various auto-immune reactions. $\mathrm{Ni}$ et al. found that M1-polarized macrophages promote degenerative phenotypes in NP cells with increased expression of key matrix catabolic genes, reduced the expression of major matrix-associated anabolic genes and upregulated transcription of inflammation-related genes [49]. Also, in a co-cultures system, Yang et al. showed that AF or NP cells exposed to macrophages upregulated the expression of pro-inflammatory mediators [50]. As for the ECM, studies have found that immunocytes could interfere with IVD ECM remodeling and downregulate aggrecan and collagen II expression [48]. In addition, the expression of matrix metalloproteinases (MMPs) and a disintegrin and metalloproteinase with thrombospondin motifs (ADAMTSs) were increased in IVD with autoimmune reaction, causing the degradation of ECM $[51,52]$. Taken together, the auto-immune reaction could stimulate immunocytes and inflammatory cytokines infiltration, and these factors could in turn impact on the IVD with harmful influence. Nevertheless, more studies are desired to explore the underlying mechanism and downstream pathways in the auto-immune response of NP tissue.

\section{Implications for clinical management of IVD degeneration}

The steady state of IVD immune privilege is fundamental to the homeostasis of the IVD. The AF and the $\mathrm{CEP}$, along with the immunosuppressive molecular factors, being regarded as the BNB, establish a strong barrier segregating the immune system from healthy IVD. However, the breakdown of the immune privilege could lead to profound consequences in IVD degeneration.

In the early stage of IVD degeneration, the breakdown of the $\mathrm{BNB}$ and the auto-immune reactions could act as a trigger with the downstream cascade reactions accelerating the pathological progress. Signs of BNB damage and NP exposure could be seen in Magnetic Resonance Imaging in early IVD degeneration. It has been indicated that Schmorl's nodes are the early stage of autoimmune response of the degenerated IVD [53]. Also, type 1 Modic change, which is widely observed in early IVD degeneration, is thought to be an autoimmune response with CEP damage [54]. Indeed, these image manifestations are strong evidence for IVD degeneration prognostication and early management [55-57]. Additionally, studies have found that inflammatory factors are upregulated in serum from IVD herniation patients. Weber et al. found that serum levels of IL-6 were significantly higher in subjects with LBP compared with control subjects [58]. Wang et al. showed that the expression of IL-10 and IL-17 was elevated in peripheral blood sera of IVD degeneration patients [59]. Most recently, Hasvik et al. found that up-regulation of circulating microRNA-17, which mediates macrophage activetion with increased TNF production, is associated with lumbar radicular pain following disc herniation [60]. Therefore, studies in the IVD auto-immune response are essential for early IVD degeneration diagnosis and management. 
A

CEP
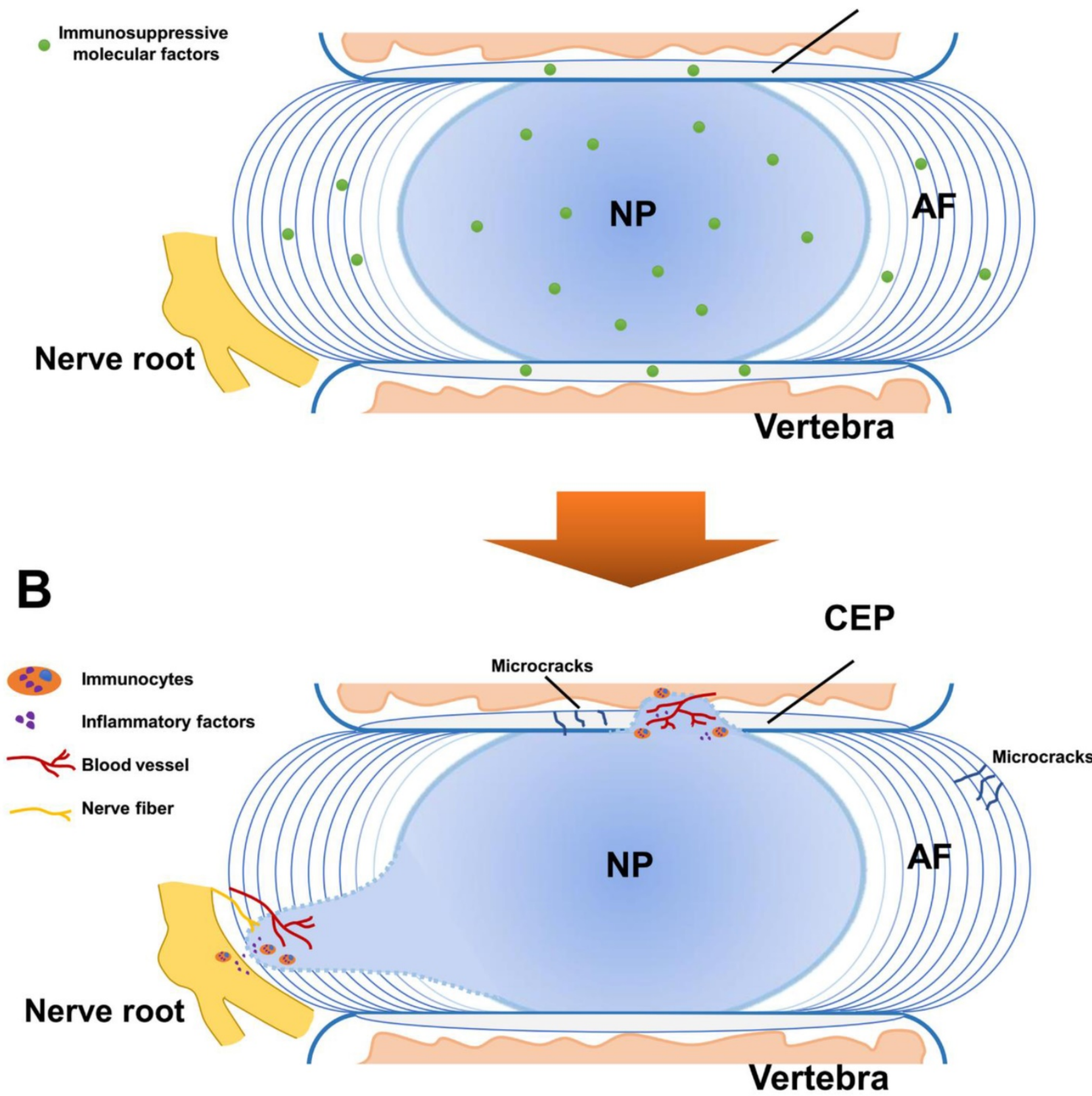

Figure 2. Schematic representation for the immune privilege of the intervertebral disc (IVD). A. In normal IVD, the blood-NP barrier (BNB) is composed of the annulus fibrosus (AF), the cartilaginous endplate (CEP) and immunosuppressive molecular factors. The BNB isolates the central nucleus pulposus (NP) from the immune system of the host and provides fundamental basis for the homeostasis of the IVD. B. The breakdown of the BNB leads to the exposure of the NP and induces auto-immune response. This effect causes immunocytes activation and inflammatory factors infiltration, contributing to the immune stress of the nerve root with vascularization and neurotization.

In the late stage of IVD degeneration, pathological changes associated with BNB damage and NP exposures are very common. These alternations include AF disruption, NP herniation and sciatica [61]. Besides physical compression, studies have strongly suggested that auto-immune reaction of the $\mathrm{NP}$ is a key mediator of radicular pain in IVD herniation [38, 42]. Studies have showed various types of activated immunocytes and inflammatory factors were recruited in NP-nerve root area [62, 63]. The immunocytes were identified including macrophages, T cells, B cells, NK cells and mast cells, and the inflammatory factors were detected such as phospholipase A2 [64], leukotrienes [65], fibroblast growth factor [66], the IL family [66], tumor necrosis factors [67], matrix metalloproteinases [68], nitric oxide [69], substance P [70], monocyte chemoattractant protein-1 [71], vascular endothelial growth factor [72] and nerve growth factor [73]. These factors constitute a complicated region contributing to the immune stress of the 
nerve root. In addition, vascularization and neurotization aggravate this situation with blood channel infiltration and nerve sensitization [74]. Therefore, strategies aiming for nerve root immunoregulation are proposed [75, 76]. In our study, we hypothesized that molecular immunotherapy might be a potential option in the treatment strategies for IVD degeneration and herniation [77].

While BNB plays an important role in normal IVD function, it is notable that not all IVD degenerations are a result of BNB failure. In specific, AF tears could lead to abnormal mechanical distribution, NP leakage and nerve ingrowth of the IVD. Additionally, microfractures in endplates which could be the result of single event trauma or part of ageing where the EP calcifies and is more prone to microfractures and exposing NP tissue to blood supply [78].

Until now, it remains a controversial topic as to the consequence of auto-immune response of IVD. While most studies indicate auto-immune response in disc herniation could be a critical factor to induce radicular pain, some studies have suggested the close relationship of auto-immune response with spontaneous regression. Komori et al. found that the disappearance of herniated NP was seen frequently in exposure to the vascular supply [79]. By examine the histological features of herniated NP tissue; Ikeda rt al. concluded that extruded or sequestrated disc was resorbed by phagocytes [80]. These studies indicate that the auto-immune response could be beneficial in some cases with exposed NP absorption. The observation of spontaneous resorption of IVD herniation has been widely reported [81-84] and the incidence of spontaneous resorption of lumbar disc herniation is reported as high as more than $60 \%$ [85]. Moreover, by addressing the molecular and cellular mechanisms involved in herniated NP regression, Cunha et al. concluded that inflammatory response could be regarded as a good prognostic indicator of spontaneous regression [86]. These studies suggested the importance of auto-immune reaction in conservative treatment for IVD herniation before final surgical involvement. Therefore, it is very important to clarify its molecular mechanism. However, the role of auto-immune reaction in this phenomenon is still unclear.

As for biological treatment for IVD degeneration, the immune privilege of the IVD cannot be ignored in stem cell transplantation and biomaterial application. In fact, stem cell could play an essential role in immune privilege rebuilding via various pathways in IVD regeneration [87]. In addition, strategies aiming to avoid auto-immune reaction should be considered in biomaterial application, as biomaterials used in
IVD regeneration are often formulated to mimic IVD composition, and usually with foreign substance.

\section{Conclusion}

The concept of IVD immune privilege arose from the apparent segregation from immune system of the body. The immune privilege state is one of the key factors to provide stable environment keeping homeostasis and normal function of the IVD. The BNB is accordingly proposed here as a complex structure, which is composed of the NP, the AF and molecular factors, and limits the penetration of the immune system. However, the breakdown of BNB could lead to auto-immune reaction of the IVD and downstream pathways. These effects play an important role in IVD degeneration process including inducement, acceleration and prognosis. Nevertheless, the study of IVD immune privilege is still limited and more studies are needed to explore the underlying mechanisms for its formation, maintenance and breakdown.

\section{Abbreviations}

ADAMTSs: a disintegrin and metalloproteinase with thrombospondin motifs; AF: annulus fibrosus; BMP: bone morphogenetic protein; BNB: blood-NP barrier; CEP: cartilaginous endplate; ECM: extracellular matrix; FasL: Fas ligand; IL: interleukin; IVD: intervertebral disc; MMPs: matrix metalloproteinases; NP: nucleus pulposus; TNF: tumor necrosis factor; VEGF: vascular endothelial growth factor.

\section{Acknowledgements}

This work was supported by grants from the Key Projects of National Natural Science Foundation of China (81730065).

\section{Competing Interests}

The authors have declared that no competing interest exists.

\section{References}

1. Roberts S, Colombier P, Sowman A, Mennan C, Rolfing JH, Guicheux J, et al. Ageing in the musculoskeletal system. Acta orthopaedica. 2016; 87: 15-25.

2. Battie MC, Joshi AB, Gibbons LE, Group IDSP. Degenerative Disc Disease: What is in a Name? Spine. 2019; 44: 1523-9.

3. Copeland B. Surgical versus nonsurgical treatment for back pain. The New England journal of medicine. 2007; 357: 1255; author reply -6.

4. Li XC, Tang Y, Wu JH, Yang PS, Wang DL, Ruan DK. Characteristics and potentials of stem cells derived from human degenerated nucleus pulposus: potential for regeneration of the intervertebral disc. BMC musculoskeletal disorders. 2017; 18: 242.

5. Tong W, Lu Z, Qin L, Mauck RL, Smith HE, Smith LJ, et al. Cell therapy for the degenerating intervertebral disc. Translational research: the journal of laboratory and clinical medicine. 2017; 181: 49-58.

6. Huang YC, Hu Y, Li Z, Luk KDK. Biomaterials for intervertebral disc regeneration: Current status and looming challenges. Journal of tissue engineering and regenerative medicine. 2018; 12: 2188-202.

7. Munir S, Rade M, Maatta JH, Freidin MB, Williams FMK. Intervertebral Disc Biology: Genetic Basis of Disc Degeneration. Current molecular biology reports. 2018; 4 : 143-50. 
8. Chen JW, Li B, Yang YH, Jiang SD, Jiang LS. Significance of hypoxia in the physiological function of intervertebral disc cells. Critical reviews in eukaryotic gene expression. 2014; 24: 193-204.

9. Roberts S, Evans H, Trivedi J, Menage J. Histology and pathology of the human intervertebral disc. The Journal of bone and joint surgery American volume. 2006; 88 Suppl 2: 10-4.

10. Capossela S, Schlafli P, Bertolo A, Janner T, Stadler BM, Potzel T, et al. Degenerated human intervertebral discs contain autoantibodies against extracellular matrix proteins. European cells \& materials. 2014; 27: 251-63; discussion 63 .

11. Wang $\mathrm{HQ}$, Samartzis D. Clarifying the nomenclature of intervertebral disc degeneration and displacement: from bench to bedside. International journal of clinical and experimental pathology. 2014; 7: 1293-8.

12. Lawson LY, Harfe BD. Developmental mechanisms of intervertebral disc and vertebral column formation. Wiley interdisciplinary reviews Developmental biology. 2017; 6 .

13. Wang F, Gao ZX, Cai F, Sinkemani A, Xie ZY, Shi R, et al. Formation, function, and exhaustion of notochordal cytoplasmic vacuoles within intervertebral disc: current understanding and speculation. Oncotarget. 2017; 8: 57800-12.

14. Boos N, Weissbach S, Rohrbach H, Weiler C, Spratt KF, Nerlich AG. Classification of age-related changes in lumbar intervertebral discs: 2002 Volvo Award in basic science. Spine. 2002; 27: 2631-44.

15. Risbud MV, Shapiro IM. Notochordal cells in the adult intervertebral disc: new perspective on an old question. Critical reviews in eukaryotic gene expression. 2011; 21: 29-41

16. Taylor AW. Ocular immune privilege. Eye. 2009; 23: 1885-9.

17. Louveau A, Harris TH, Kipnis J. Revisiting the Mechanisms of CNS Immune Privilege. Trends in immunology. 2015; 36: 569-77.

18. Zhao S, Zhu W, Xue S, Han D. Testicular defense systems: immune privilege and innate immunity. Cellular \& molecular immunology. 2014; 11: 428-37.

19. Piccinni MP. Role of immune cells in pregnancy. Autoimmunity. 2003; 36: 1-4

20. Hee HT, Chuah YJ, Tan BH, Setiobudi T, Wong HK. Vascularization and morphological changes of the endplate after axial compression and distraction of the intervertebral disc. Spine. 2011; 36: 505-11.

21. Johnson WE, Caterson B, Eisenstein SM, Roberts S. Human intervertebral disc aggrecan inhibits endothelial cell adhesion and cell migration in vitro. Spine. 2005; 30: 1139-47.

22. Takada T, Nishida K, Doita M, Kurosaka M. Fas ligand exists on intervertebral disc cells: a potential molecular mechanism for immune privilege of the disc. Spine. 2002; 27: 1526-30.

23. Kaneyama S, Nishida K, Takada T, Suzuki T, Shimomura T, Maeno K, et al. Fas ligand expression on human nucleus pulposus cells decreases with disc degeneration processes. Journal of orthopaedic science : official journal of the Japanese Orthopaedic Association. 2008; 13: 130-5.

24. Sun Z, Wan ZY, Guo YS, Wang HQ, Luo ZJ. FasL on human nucleus pulposus cells prevents angiogenesis in the disc by inducing Fas-mediated apoptosis of vascular endothelial cells. International journal of clinical and experimental pathology. 2013; 6: 2376-85.

25. Liu ZH, Sun Z, Wang HQ, Ge J, Jiang TS, Chen YF, et al. FasL expression on human nucleus pulposus cells contributes to the immune privilege of intervertebral disc by interacting with immunocytes. International journal of medical sciences. 2013; 10: 1053-60.

26. Wiet MG, Piscioneri A, Khan SN, Ballinger MN, Hoyland JA, Purmessur D. Mast Cell-Intervertebral disc cell interactions regulate inflammation, catabolism and angiogenesis in Discogenic Back Pain. Scientific reports. 2017; 7: 12492.

27. Bach FC, Tellegen AR, Beukers M, Miranda-Bedate A, Teunissen M, de Jong WAM, et al. Biologic canine and human intervertebral disc repair by notochordal cell-derived matrix: from bench towards bedside. Oncotarget. 2018; 9: 26507-26.

28. Kim JH, Moon HJ, Lee JH, Kim JH, Kwon TH, Park YK. Rabbit notochordal cells modulate the expression of inflammatory mediators by human annulus fibrosus cells cocultured with activated macrophage-like THP-1 cells. Spine. 2012; 37: 1856-64.

29. Cornejo MC, Cho SK, Giannarelli C, Iatridis JC, Purmessur D. Soluble factors from the notochordal-rich intervertebral disc inhibit endothelial cell invasion and vessel formation in the presence and absence of pro-inflammatory cytokines. Osteoarthritis and cartilage. 2015; 23: 487-96.

30. Kwon WK, Moon HJ, Kwon TH, Park YK, Kim JH. Influence of rabbit notochordal cells on symptomatic intervertebral disc degeneration: anti-angiogenic capacity on human endothelial cell proliferation under hypoxia. Osteoarthritis and cartilage. 2017; 25: 1738-46

31. Purmessur D, Cornejo MC, Cho SK, Roughley PJ, Linhardt RJ, Hecht AC, et al. Intact glycosaminoglycans from intervertebral disc-derived notochordal cell-conditioned media inhibit neurite growth while maintaining neuronal cell viability. The spine journal : official journal of the North American Spine Society. 2015; 15: 1060-9.

32. de Vries SAH, van Doeselaar M, Meij BP, Tryfonidou MA, Ito K. Notochordal cell matrix: An inhibitor of neurite and blood vessel growth? Journal of orthopaedic research : official publication of the Orthopaedic Research Society. 2018; 36: 3188-95

33. Vernon-Roberts B, Moore RJ, Fraser RD. The natural history of age-related disc degeneration: the pathology and sequelae of tears. Spine. 2007; 32: 2797-804.
34. Yasuma T, Makino E, Saito S, Inui M. Histological development of intervertebral disc herniation. The Journal of bone and joint surgery American volume. 1986; 68: 1066-72.

35. Stefanakis M, Al-Abbasi M, Harding I, Pollintine P, Dolan P, Tarlton J, et al. Annulus fissures are mechanically and chemically conducive to the ingrowth of nerves and blood vessels. Spine. 2012; 37: 1883-91.

36. Gertzbein SD, Tait JH, Devlin SR. The stimulation of lymphocytes by nucleus pulposus in patients with degenerative disk disease of the lumbar spine. Clinical orthopaedics and related research. 1977: 149-54

37. Bobechko WP, Hirsch C. Auto-Immune Response To Nucleus Pulposus In the Rabbit. The Journal of bone and joint surgery British volume. 1965; 47: 574-80.

38. Kawakami M, Tamaki T, Weinstein JN, Hashizume H, Nishi H, Meller ST. Pathomechanism of pain-related behavior produced by allografts of intervertebral disc in the rat. Spine. 1996; 21: 2101-7.

39. Satoh K, Konno S, Nishiyama K, Olmarker K, Kikuchi S. Presence and distribution of antigen-antibody complexes in the herniated nucleus pulposus. Spine. 1999; 24: 1980-4

40. Geiss A, Larsson K, Rydevik B, Takahashi I, Olmarker K. Autoimmune properties of nucleus pulposus: an experimental study in pigs. Spine. 2007; 32: $168-73$.

41. Geiss A, Larsson K, Junevik K, Rydevik B, Olmarker K. Autologous nucleus pulposus primes $\mathrm{T}$ cells to develop into interleukin-4-producing effector cells: an experimental study on the autoimmune properties of nucleus pulposus. Journal of orthopaedic research : official publication of the Orthopaedic Research Society. 2009; 27: 97-103.

42. Murai K, Sakai D, Nakamura Y, Nakai T, Igarashi T, Seo N, et al. Primary immune system responders to nucleus pulposus cells: evidence for immune response in disc herniation. European cells \& materials. 2010; 19: 13-21.

43. Geiss A, Sobottke R, Stefan Delank K, Eysel P. Macrophages do not represent the main cell type in sequestrated and extruded intervertebral discs: evidence for their involvement in disc resorption, rather than initiation of an immune response. Global Spine Journal. 2014; 4:1

44. Lee S, Millecamps M, Foster DZ, Stone LS. Long-term histological analysis of innervation and macrophage infiltration in a mouse model of intervertebral disc injury-induced low back pain. Journal of orthopaedic research : official publication of the Orthopaedic Research Society. 2019.

45. Monchaux M, Forterre S, Spreng D, Karol A, Forterre F, Wuertz-Kozak K. Inflammatory Processes Associated with Canine Intervertebral Disc Herniation. Frontiers in immunology. 2017; 8: 1681

46. Takada T, Nishida K, Maeno K, Kakutani K, Yurube T, Doita M, et al. Intervertebral disc and macrophage interaction induces mechanical hyperalgesia and cytokine production in a herniated disc model in rats. Arthritis and rheumatism. 2012; 64: 2601-10.

47. Stich S, Stolk M, Girod PP, Thome C, Sittinger M, Ringe J, et al. Regenerative and immunogenic characteristics of cultured nucleus pulposus cells from human cervical intervertebral discs. PloS one. 2015; 10: e0126954.

48. Silva AJ, Ferreira JR, Cunha C, Corte-Real JV, Bessa-Goncalves M, Barbosa MA, et al. Macrophages Down-Regulate Gene Expression of Intervertebral Disc Degenerative Markers Under a Pro-inflammatory Microenvironment. Frontiers in immunology. 2019; 10: 1508.

49. Ni L, Zheng Y, Gong T, Xiu C, et al. Proinflammatory macrophages promote degenerative phenotypes in rat nucleus pulpous cells partly through ERK and JNK signaling. Journal of cellular physiology. 2019; 234: 5362-71.

50. Yang H, Liu B, Liu Y, He D, Xing Y, An Y, et al. Secreted Factors From Intervertebral Disc Cells and Infiltrating Macrophages Promote Degenerated Intervertebral Disc Catabolism. Spine. 2019; 44: E520-E9.

51. Hwang MH, Kim KS, Yoo CM, Shin JH, Nam HG, Jeong JS, et al. Photobiomodulation on human annulus fibrosus cells during the intervertebral disk degeneration: extracellular matrix-modifying enzymes. Lasers in medical science. 2016; 31: 767-77.

52. Tsuru M, Nagata K, Ueno T, Jimi A, Irie K, Yamada A, et al. Electron microscopic observation of established chondrocytes derived from human intervertebral disc hernia (KTN-1) and role of macrophages in spontaneous regression of degenerated tissues. The spine journal : official journal of the North American Spine Society. 2001; 1: 422-31

53. Zhang N, Li FC, Huang YJ, Teng C, Chen WS. Possible key role of immune system in Schmorl's nodes. Medical hypotheses. 2010; 74: 552-4.

54. Dudli S, Liebenberg E, Magnitsky S, Lu B, Lauricella M, Lotz JC. Modic type 1 change is an autoimmune response that requires a proinflammatory milieu provided by the 'Modic disc'. The spine journal : official journal of the North American Spine Society. 2018; 18: 831-44.

55. Lan M, Ou Y, Wang C, Wei W, Lu X, Wei J, et al. Patients with Modic type 2 change have a severe radiographic representation in the process of lumbar degeneration: a retrospective imaging study. Journal of orthopaedic surgery and research. 2019; 14: 298

56. Wan J, Zhang XS. Pre-operative blood test for antibody to nucleus pulposus may distinguish types of lumbar disc herniation. Medical hypotheses. 2010; 75: $464-5$

57. Sahoo MM, Mahapatra SK, Kaur S, Sarangi J, Mohapatra M. Significance of Vertebral Endplate Failure in Symptomatic Lumbar Disc Herniation. Global spine journal. 2017; $7: 230-8$.

58. Weber KT, Alipui DO, Sison CP, Bloom O, Quraishi S, Overby MC, et al. Serum levels of the proinflammatory cytokine interleukin- 6 vary based on diagnoses in individuals with lumbar intervertebral disc diseases. Arthritis research \& therapy. 2016; 18: 3 . 
59. Wang S, Wei J, Fan Y, Ding H, Tian H, Zhou X, et al. Progranulin Is Positively Associated with Intervertebral Disc Degeneration by Interaction with IL-10 and IL-17 Through TNF Pathways. Inflammation. 2018; 41: 1852-63.

60. Hasvik E, Schjolberg T, Jacobsen DP, Haugen AJ, Grovle L, Schistad EI, et al. Up-regulation of circulating microRNA-17 is associated with lumbar radicular pain following disc herniation. Arthritis research \& therapy. 2019; 21: 186.

61. van Tulder M, Peul W, Koes B. Sciatica: what the rheumatologist needs to know. Nature reviews Rheumatology. 2010; 6: 139-45.

62. Kokubo Y, Uchida K, Kobayashi S, Yayama T, Sato R, Nakajima H, et al. Herniated and spondylotic intervertebral discs of the human cervical spine: histological and immunohistological findings in 500 en bloc surgical samples. Laboratory investigation. Journal of neurosurgery Spine. 2008; 9: 285-95.

63. Risbud MV, Shapiro IM. Role of cytokines in intervertebral disc degeneration: pain and disc content. Nature reviews Rheumatology. 2014; 10: 44-56.

64. Korovessis PG. Phospholipase A2 activity in herniated lumbar discs. Spine. 1999; $24: 99$

65. Kawakami M, Matsumoto T, Tamaki T. Roles of thromboxane A2 and leukotriene B4 in radicular pain induced by herniated nucleus pulposus. Journal of orthopaedic research : official publication of the Orthopaedic Research Society. 2001; 19: 472-7.

66. Xu Z, Zhou X, Chen G. Expression and Mechanism of Interleukin 1 (IL-1) Interleukin 2 (IL-2), Interleukin 8 (IL-8), BMP, Fibroblast Growth Factor 1 (FGF1), and Insulin-Like Growth Factor (IGF-1) in Lumbar Disc Herniation. Medical science monitor : international medical journal of experimental and clinical research. 2019; 25: 984-90

67. Johnson ZI, Schoepflin ZR, Choi H, Shapiro IM, Risbud MV. Disc in flames: Roles of TNF-alpha and IL-1beta in intervertebral disc degeneration. European cells \& materials. 2015; 30: 104-16; discussion 16-7.

68. Ozkanli S, Kaner T, Efendioglu M, Basaran R, Senol M, Zemheri E, et al. The relation of matrix metalloproteinase 1, 2, 3 expressions with clinical and radiological findings in primary and recurrent lumbar disc herniations. Turkish neurosurgery. 2015; 25: 111-6.

69. Furusawa N, Baba H, Miyoshi N, Maezawa Y, Uchida K, Kokubo Y, et al. Herniation of cervical intervertebral disc: immunohistochemical examination and measurement of nitric oxide production. Spine. 2001; 26: 1110-6.

70. Palmgren T, Gronblad M, Virri J, Seitsalo S, Ruuskanen M, Karaharju E. Immunohistochemical demonstration of sensory and autonomic nerve terminals in herniated lumbar disc tissue. Spine. 1996; 21: 1301-6.

71. Yoshida M, Nakamura T, Sei A, Kikuchi T, Takagi K, Matsukawa A. Intervertebral disc cells produce tumor necrosis factor alpha, interleukin-1beta, and monocyte chemoattractant protein-1 immediately after herniation: an experimental study using a new hernia model. Spine. 2005; 30: 55-61.

72. Tsarouhas A, Soufla G, Tsarouhas K, Katonis P, Pasku D, Vakis A, et al. Molecular profile of major growth factors in lumbar intervertebral disc herniation: Correlation with patient clinical and epidemiological characteristics. Molecular medicine reports. 2017; 15: 2195-203.

73. Aoki $\mathrm{Y}$, Nakajima A, Ohtori S, Takahashi $\mathrm{H}$, Watanabe $\mathrm{F}$, Sonobe $\mathrm{M}$, et al. Increase of nerve growth factor levels in the human herniated intervertebral disc: can annular rupture trigger discogenic back pain? Arthritis research \& therapy. 2014; 16: R159.

74. Lama P, Le Maitre CL, Harding IJ, Dolan P, Adams MA. Nerves and blood vessels in degenerated intervertebral discs are confined to physically disrupted tissue. Journal of anatomy. 2018; 233: 86-97.

75. Suyama K, Sakai D, Hirayama N, Nakamura Y, Matsushita E, Terayama $H_{\text {, et }}$ al. Effects of interleukin-17A in nucleus pulposus cells and its small-molecule inhibitors for intervertebral disc disease. Journal of cellular and molecular medicine. 2018; 22: 5539-51.

76. Di Martino A, Merlini L, Faldini C. Autoimmunity in intervertebral disc herniation: from bench to bedside. Expert opinion on therapeutic targets. 2013; 17: 1461-70.

77. Sun Z, Liu ZH, Chen YF, Zhang YZ, Wan ZY, Zhang WL, et al. Molecular immunotherapy might shed a light on the treatment strategies for disc degeneration and herniation. Medical hypotheses. 2013; 81: 477-80.

78. Adams MA. Biomechanics of back pain. Acupuncture in medicine : journal of the British Medical Acupuncture Society. 2004; 22: 178-88.

79. Komori H, Shinomiya K, Nakai O, Yamaura I, Takeda S, Furuya K. The natural history of herniated nucleus pulposus with radiculopathy. Spine. 1996; 21: 225-9.

80. Ikeda T, Nakamura T, Kikuchi T, Umeda S, Senda H, Takagi K. Pathomechanism of spontaneous regression of the herniated lumbar disc: histologic and immunohistochemical study. Journal of spinal disorders. 1996; 9: $136-40$

81. Orief T, Orz Y, Attia W, Almusrea K. Spontaneous resorption of sequestrated intervertebral disc herniation. World neurosurgery. 2012; 77: 146-52.

82. Cvetanovich GL, Hsu AR, Frank RM, An HS, Andersson GB. Spontaneous resorption of a large cervical herniated nucleus pulposus. American journal of orthopedics. 2014; 43: E140-5.

83. Liu JT, Li XF, Yu PF, Li XC, Qian O, Liu GH, et al. Spontaneous resorption of a large lumbar disc herniation within 4 months. Pain physician. 2014; 17: E803-6.

84. Turk O, Yaldiz C. Spontaneous regression of cervical discs: Retrospective analysis of 14 cases. Medicine. 2019; 98: e14521.

85. Zhong M, Liu JT, Jiang H, Mo W, Yu PF, Li XC, et al. Incidence of Spontaneous Resorption of Lumbar Disc Herniation: A Meta-Analysis. Pain physician. 2017; 20: E45-E52.
86. Cunha $\mathrm{C}$, Silva AJ, Pereira P, Vaz R, Goncalves RM, Barbosa MA. The inflammatory response in the regression of lumbar disc herniation. Arthritis research \& therapy. 2018; 20: 251.

87. Ma CJ, Liu X, Che L, Liu ZH, Samartzis D, Wang HQ. Stem Cell Therapies for Intervertebral Disc Degeneration: Immune Privilege Reinforcement by Fas/FasL Regulating Machinery. Current stem cell research \& therapy. 2015; 10: $285-95$ 